\title{
The Heat Flux Vector(s) in a Two Component Fluid Mixture
}

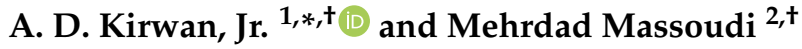 \\ 1 School of Marine Science and Policy, University of Delaware, 103 Robinson Hall, Newark, DE 19716, USA \\ 2 U.S. Department of Energy, National Energy Technology Laboratory (NETL), 626 Cochrans Mill Road, \\ P.O. Box 10940, Pittsburgh, PA 15236, USA; massoudi@netl.doe.gov \\ * Correspondence: adk@udel.edu; Tel.: +1-302-831-2977 \\ + Both authors contributed equally to this work.
}

Received: 22 January 2020; Accepted: 12 May 2020; Published: 20 May 2020

\begin{abstract}
Bulk kinematic properties of mixtures such as velocity are known to be the density weighed averages of the constituent velocities. No such paradigm exists for the heat flux of mixtures when the constituents have different temperatures. Using standard principles such as frame indifference, we address this topic by developing linear constitutive equations for the constituent heat fluxes, the interaction force between constituents, and the stresses for a mixture of two fluids. Although these equations contain 18 phenomenological coefficients, we are able to use the Clausius-Duhem inequality to obtain inequalities involving the principal and cross flux coefficients. The theory is applied to some special cases and shown to reduce to standard results when the constituents have the same temperature.
\end{abstract}

Keywords: mixtures; Fourier's heat conduction; heat flux vector; continuum theory; Clausius-Duhem inequality

PACS: J0101

\section{Introduction}

Of general concern here are the heat fluxes of multicomponent materials where the temperatures of the components are not equal. A general theory for multi-temperature mixtures was proposed by Dunwoody and Müller [1], extended by Bowen and Garcia [2], and applied to a mixture of Navier Stokes fluids by Ahmadi [3]. Despite this theoretical framework, as well as many examples from both the environment and industry of multicomponent materials with distinct constituent temperatures, there is a paucity of research on the thermodynamic implications of characterizing the bulk properties of the heat fluxes of such materials. Perhaps this is due to the myth that such materials quickly come to thermal equilibrium. In reality, many processes such as radiative heat transfer, disparate thermal conductivities of constituent materials, and relative motion of the constituents may disrupt a rapid approach to overall thermal equilibrium.

Quantification of the heat flux has been a goal of researchers ever since the beginning of thermodynamics. Early theories were phenomenologically motivated, but in recent years considerable attention has been devoted to Maxwell-Cattaneo type models; see [4,5] and a more recent discussion in Jou et al. [6]. Typically this approach leads to some form of the telegraph equation, a special case of which is Fourier's law of heat conduction. As our concern here is not with the propagation of heat pulses but with constraints imposed on constitutive models by the second law, it is sufficient to focus here on Fourier's law. For an isotropic material this is given by the familiar expression:

$$
\mathbf{q}=-k \nabla \theta
$$


Here $\mathbf{q}$ is the heat flux vector, $\theta$ is the temperature, $\nabla$ denotes the gradient operator, and $k$ is a material property known as thermal conductivity. The latter is readily obtained from standard measurements of $\nabla \theta$ and $\mathbf{q}$.

However, a substance composed of two components with different thermal conductivities is not so trivial. Consider two scenarios of a mixture of two constituents, one with temperature $\theta_{a}$ and thermal conductivity $k_{a}$ and the other with temperature $\theta_{b}$ and thermal conductivity $k_{b}$. If both follow the Fourier's heat conduction relation and the two components are at thermal equilibrium, i.e., $\theta_{a}=\theta_{b}=\theta$, then

$$
\begin{aligned}
& \mathbf{q}_{a}=-k_{a} \nabla \theta \\
& \mathbf{q}_{b}=-k_{b} \nabla \theta .
\end{aligned}
$$

One is then tempted to take as the mixture heat flux

$$
\mathbf{q}_{\text {mix }}=\mathbf{q}_{a}+\mathbf{q}_{b}=-\left(k_{a}+k_{b}\right) \nabla \theta=-k_{\text {mix }} \nabla \theta .
$$

This is just like the single constituent case, and Fourier's law applies to the mixture. Standard methods can determine the conductivity of the mixture.

Now suppose the two constituents are at different temperatures. Allowing for cross-thermal gradient heat fluxes one has

$$
\begin{aligned}
& \mathbf{q}_{a}=-k_{a a} \nabla \theta_{a}-k_{a b} \nabla \theta_{b} \\
& \mathbf{q}_{b}=-k_{b a} \nabla \theta_{b}-k_{b b} \nabla \theta_{b} .
\end{aligned}
$$

Simply adding the fluxes as in the previous case gives

$$
\mathbf{q}_{\text {mix }}=-\left(k_{a a}+k_{b a}\right) \nabla \theta_{a}-\left(k_{a b}+k_{b b}\right) \nabla \theta_{b} .
$$

Although the definition of the mixture heat flux is the same, it is not possible to prescribe both a unique temperature and conductivity for the mixture if the constituents are not in thermal equilibrium.

These idealized examples raise the ontological question: What flux laws are appropriate that ensure consistency with the primitive notion that the consequent flow of heat does not violate the second law? In this regard Petroski [7] pointed out that for many non-homogenous and anisotropic materials the simple Fourier's assumption does not hold (see also [8]). For complex materials such as polymers, granular materials, and non-Newtonian fluids, the thermal conductivity of the material is assumed to depend on parameters such as volume fraction, particle size, shear rate, etc. [9-11]. Also see [12,13] for detailed reviews. These and other matters have spurred research on general theories such as Maxwell-Cattaneo models, as noted previously.

To address the ontological question noted above, we develop a unified theory for multicomponent materials with different temperatures. Previously, Massoudi [14] used a mixture theory approach to obtain an explicit constitutive relation for the heat flux vector(s) $\mathbf{q}$ of a two-phase mixture with a single temperature. We extend that study to allow for distinct temperatures $\theta_{1}$ and $\theta_{2}$ for the components.

We intend for this study to provide some theoretical guidance for experimenters attempting to establish the phenomenological parameters for mixtures. To achieve this we utilize the second law requirement that the rate of entropy production of the mixture as a whole is non-negative to establish inequality constraints on the phenomenological coefficients. In this regard some investigators have also imposed Onsager's reciprocal relations to reduce the number of cross flux coefficients. For example in (4) this constraint requires $k_{a b}=k_{b a}$, thus reducing the number of coefficients that are needed to be determined to three. By appealing to just the second law, we do not need to make this restriction. In this regard it should be noted that in addition to Onsager type constraints, Klika et al. [15] and Klika and Krause [16] have developed functional constraints for phenomenological coefficients. Although their theory does not necessarily reduce the number of coefficients, it should simplify experimental procedures necessary to establish their values. 
Our report is organized as follows. Section 2 gives a succinct review of the conservation equations for mass, linear momentum, angular momentum, and energy, and the entropy tendency equation for the entropy for a two-component fluid mixture. The traditional approach introduces the Helmholtz free energy into the Clausius-Duhem inequality from which constraints on phenomenological coefficients can be developed from constitutive equations with explicit statements connecting the fluxes and thermodynamic forces, i.e., rate variables. Our observation is that thermodynamic constraints arising from the inequality sometimes are neglected in constitutive theories for nonlinear and complex materials such as polymers and second grade fluids. To address this and other more fundamental issues Rajagopal and Srinivasa [17-19] developed a consistent theory built around constitutive equations where the thermodynamic forces and fluxes are connected through implicit relations.

As there is a substantial body of literature on applications of the traditional framework to mixture theory we elect to follow that approach. However, we deviate slightly from that approach by relying on classical irreversible thermodynamics concepts [20] and utilize the Gibbs relation for the mixture to establish the non-negative entropy production. In Section 3 we develop linear constitutive relations for this two-component mixture. We extend previous work to allow for constituents with different temperatures. Section 4 addresses constraints imposed on the constitutive equations by the second law of thermodynamics. Section 5 analyzes some special cases and implications for characterizing the heat flux of multi-temperature mixtures. We conclude in section 6 with a discussion of the implications of this study.

\section{Review of the Basic Equations of Mixture Theory}

\subsection{Background}

Foundations of mixture theory, sometimes called the theory of interacting continua, are given in books by [21,22], and early review articles by [23-25]. A recent review article by Klika, [20], provides a critical assessment of standard approaches to multicomponent material. Here we appeal to the classical irreversible thermodynamics paradigm as advocated by [20]. The conceptual premise of this approach regards each component as a single continuum, denoted as $S_{\alpha}$, and at each instant of time, every point in space is considered to be occupied by a particle belonging to each component of the mixture. Conservation laws are then written for each component accounting for interactions with other constituents. Constitutive relations for the fluxes of heat and momentum are used to close the governing equations.

Balance equations for the conservation of mass, linear momentum, angular momentum, and energy equations for each constituent are obtained by summing the appropriate constituent equations over all constituents; see for example [23,25]. Many early researchers attributed fundamental significance to the mixture equations, but more recent works simply regard these as statements that the mixture obeys the basic principles of physics. See $[20,26,27]$ for additional discussion of this matter.

Our concern here is with the mixture of the two constituents that comprise a thermo-mechanical system in which electromagnetic and chemical reactions are ignored and material isotropy is assumed. Of course, the latter effects can be incorporated in mixture theory but are not essential here.

At each instant of time $t$, it is assumed that each point in space is occupied by particles belonging to both $S_{1}$ and $S_{2}$. Then the equations describing the motion of a two-component system are (see $[20,23,24,28]$ ):

$$
\mathbf{x}_{1}=\mathcal{X}_{1}\left(\mathbf{X}_{1}, t\right), \mathbf{x}_{2}=\mathcal{X}_{2}\left(\mathbf{X}_{2}, t\right)
$$

while the kinematical quantities associated with these motions are

$$
\begin{gathered}
\mathbf{v}_{1}=\frac{d_{1} \mathbf{x}_{1}}{d t}, \mathbf{v}_{2}=\frac{d_{2} \mathbf{x}_{2}}{d t}, \\
\mathbf{D}_{1}=\frac{1}{2}\left(\nabla \mathbf{v}_{1}+\left(\nabla \mathbf{v}_{1}\right)^{T}\right), \mathbf{D}_{2}=\frac{1}{2}\left(\nabla \mathbf{v}_{2}+\left(\nabla \mathbf{v}_{2}\right)^{T}\right),
\end{gathered}
$$




$$
\mathbf{W}_{1}=\frac{1}{2}\left(\nabla \mathbf{v}_{1}-\left(\nabla \mathbf{v}_{1}\right)^{T}\right), \mathbf{W}_{2}=\frac{1}{2}\left(\nabla \mathbf{v}_{2}-\left(\nabla \mathbf{v}_{2}\right)^{T}\right) .
$$

In the above, $\mathbf{v}$ is the velocity field, $\mathbf{D}$ is the symmetric part of velocity gradient or deformation rate tensor, $\mathbf{W}$ is the skew-symmetric part of the velocity gradient or spin tensor, the superscript $T$ indicates the matrix transpose, and $\frac{d_{1}}{d t}$ and $\frac{d_{2}}{d t}$ denote differentiation with respect to time holding $\mathbf{X}_{1}$ and $\mathbf{X}_{2}$ fixed, respectively.

The densities $\rho_{1}$ and $\rho_{2}$ for these two components are

$$
\rho_{1}=\phi \rho_{10}, \rho_{2}=(1-\phi) \rho_{20}
$$

where $\rho_{10}$ and $\rho_{20}$ are the pristine densities of the constituents in the reference configuration; $\phi$ is the volume fraction of constituent 1 . In the case of a mixture of a fluid and solid, $\phi$ usually refers to the solid phase. Generally, $0 \leq \phi<\phi_{\max }<1$. The function $\phi$ is represented as a continuous function of position and time; in reality, $\phi$ is either one or zero at any position and at any time, depending upon whether one is pointing to a particle in constituent 1 or 2 at that position. In other words, the real volume distribution content has been averaged, in some sense, over a neighborhood of any given position. In practice, $\phi$ has a maximum value, especially for fluid-solid particle systems, generally designated as the maximum packing fraction, which depends on the shape, size, method of packing, etc.

The mixture density $\rho$ is given by

$$
\rho=\rho_{1}+\rho_{2}
$$

and a mixture or average velocity $\mathbf{v}$ typically is defined by

$$
\rho \mathbf{v}=\rho_{1} \mathbf{v}_{1}+\rho_{2} \mathbf{v}_{2} \text {. }
$$

Of course other weighting functions could be used in (12), but we prefer the partial densities since momentum density is fundamental in the equations of motion.

\subsection{Conservation of Mass}

Assuming no chemical reactions involving mass exchange between the constituents, the equations of conservation of mass for each constituent in the Eulerian form are (see [20,22-24]):

$$
\begin{aligned}
& \frac{d_{1} \rho_{1}}{d t}+\rho_{1} \nabla \cdot \mathbf{v}_{1}=\frac{\partial \rho_{1}}{\partial t}+\nabla \cdot\left(\rho_{1} \mathbf{v}_{1}\right)=0 \\
& \frac{d_{2} \rho_{2}}{d t}+\rho_{2} \nabla \cdot \mathbf{v}_{2}=\frac{\partial \rho_{2}}{\partial t}+\nabla \cdot\left(\rho_{2} \mathbf{v}_{2}\right)=0
\end{aligned}
$$

where $\frac{\partial}{\partial t}$ is the partial derivative with respect to time holding the space dimensions fixed. Using (11) and (12) it is clear that

$$
\frac{\partial \rho}{\partial t}+\nabla \cdot(\rho \mathbf{v})=0
$$

\subsection{Conservation of Linear Momentum}

Let $\mathbf{T}_{1}$ and $\mathbf{T}_{2}$ denote the respective partial stress tensors. We follow Eckart [29] and include hydrostatic or equilibrium components in these stresses. Then the balances of linear momentum for the two components are given by

$$
\begin{aligned}
& \rho_{1} \frac{d_{1} \mathbf{v}_{1}}{d t}=\nabla \cdot \mathbf{T}_{1}+\rho_{1} \mathbf{b}_{1}+\mathbf{f}_{I} \\
& \rho_{1} \frac{d_{2} \mathbf{v}_{2}}{d t}=\nabla \cdot \mathbf{T}_{2}+\rho_{2} \mathbf{b}_{2}-\mathbf{f}_{I} .
\end{aligned}
$$

Here $\mathbf{b}_{\alpha}$ are the external body forces and $\mathbf{f}_{I}$ is a frame invariant form of the interaction forces resulting in the exchange of momentum between the components. The importance of frame invariance in this context is discussed by Truesdell and Noll [30]. Note that in writing (14) we appealed to Newton's third 
law of motion to require that the latter exactly cancels in the conservation of total linear momentum of the mixture.

Once the partial stress tensors are specified, a mixture stress tensor can be defined as in [31,32].

$$
\mathbf{T}_{\text {mix }}=\mathbf{T}_{1}+\mathbf{T}_{2}
$$

where, as suggested by [33],

$$
\begin{aligned}
& \mathbf{T}_{1}=(1-\phi) \mathbf{T}_{10} \\
& \mathbf{T}_{2}=\phi \mathbf{T}_{20} .
\end{aligned}
$$

Here $\mathbf{T}_{10}$ and $\mathbf{T}_{20}$ are the pristine stress tensors for the respective material. Thus the mixture stress tensor reduces to that of substance 1 as $\phi \rightarrow 0$ and to that of substance 2 as $\phi \rightarrow 1$. We note that there are other ways to define $\mathbf{T}_{m i x}$ in terms of $\mathbf{T}_{1}$ and $\mathbf{T}_{2}$, such as equation (2.13) in [34].

\subsection{Conservation of Angular Momentum}

The balance of moment of momentum for mixtures was analyzed in some detail by Bowen, [25]. It is sufficient here to write this as

$$
\begin{aligned}
& \mathbf{T}_{\alpha}=\mathbf{T}_{\alpha}^{T}+\mathbf{M}_{\alpha}, \alpha=1,2 \\
& \mathbf{M}_{1}+\mathbf{M}_{2}=0
\end{aligned}
$$

Bowen [25] refers to $\mathbf{M}_{\alpha}$ as the couple stress for constituent $\alpha$. In the absence of couple stresses, i.e., when $\mathbf{M}_{\alpha}=0$ then (17) states the total stress tensor is symmetric. Equation (17) states that the constituent couple stresses cancel each other.

\subsection{Conservation of Energy}

Each component of the mixture satisfies a balance of energy equation. This is expressed as

$$
\begin{aligned}
& \rho_{1} \frac{d_{1}}{d t}\left(u_{1}+\frac{\mathbf{v}_{1} \cdot \mathbf{v}_{1}}{2}\right)=\nabla \cdot\left(\mathbf{T}_{1} \cdot \mathbf{v}_{1}-\mathbf{q}_{1}\right)+\rho_{1} r_{1}+\mathbf{v}_{1} \cdot\left(\rho_{1} \mathbf{b}_{1}+\mathbf{f}_{I}\right)+e_{1} \\
& \rho_{1} \frac{d_{2}}{d t}\left(u_{2}+\frac{\mathbf{v}_{2} \cdot \mathbf{v}_{2}}{2}\right)=\nabla \cdot\left(\mathbf{T}_{2} \cdot \mathbf{v}_{2}-\mathbf{q}_{2}\right)+\rho_{2} r_{2}+\mathbf{v}_{2} \cdot\left(\rho_{2} \mathbf{b}_{2}-\mathbf{f}_{I}\right)+e_{2} .
\end{aligned}
$$

Here the $u_{\alpha}$ denote the specific internal energies of constituent $\alpha, \mathbf{q}_{\alpha}$ is the partial heat flux of $\alpha, r_{\alpha}$ accounts for the external heat supply of $\alpha$, and $e_{\alpha}$ is the frame invariant energy supply to $\alpha$.

For our purposes, it is more useful to focus on the internal energy balances for each constituent. This is obtained by subtracting the kinetic energy equations derived from (14). Thus

$$
\begin{aligned}
& \rho_{1} \frac{d_{1} u_{1}}{d t}=\mathbf{T}_{1}: \nabla \mathbf{v}_{1}-\nabla \cdot \mathbf{q}_{1}+\rho_{1} r_{1}+e_{1} \\
& \rho_{2} \frac{d_{2} u_{2}}{d t}=\mathbf{T}_{2}: \nabla \mathbf{v}_{2}-\nabla \cdot \mathbf{q}_{2}+\rho_{2} r_{2}+e_{2} .
\end{aligned}
$$

Following [25] we use (8), (9), and (17) to express (19) as

$$
\begin{aligned}
& \rho_{1} \frac{d_{1} u_{1}}{d t}=\mathbf{T}_{1}^{T}: \mathbf{D}_{1}-\nabla \cdot \mathbf{q}_{1}+\rho_{1} r_{1}+e_{1}-\left(\frac{1}{2}\right) \mathbf{M}_{1}: \mathbf{W}_{1} \\
& \rho_{2} \frac{d_{2} u_{2}}{d t}=\mathbf{T}_{2}^{T}: \mathbf{D}_{2}-\nabla \cdot \mathbf{q}_{2}+\rho_{2} r_{2}+e_{2}-\left(\frac{1}{2}\right) \mathbf{M}_{2}: \mathbf{W}_{2} .
\end{aligned}
$$

As shown in [25], Equation (20) is frame invariant if the internal energy sources $e_{\alpha}$ include the work performed by the $\mathbf{M}_{\alpha}$. There the energy sources are written as 


$$
e_{\alpha}-\left(\frac{1}{2}\right) \mathbf{M}_{\alpha}: \mathbf{W}_{\alpha}=e_{\alpha}^{*}
$$

Of course there may be additional internal energy sources. One important supply relevant to the heat flux is the heat generated by the friction resulting from the relative motion of the constituents. In Appendix 5A of [21] Bowen writes this as

$$
\mathcal{W}=\left(\mathbf{v}_{1}-\mathbf{v}_{2}\right) \cdot \mathbf{f}_{I}
$$

Thus we specify objective internal energy supplies as

$$
\begin{aligned}
& e_{1}^{*}=-\left[\left(\frac{1}{2}\right) \mathbf{M}_{1}: \mathbf{W}_{1}+\mathcal{W}\right] \\
& e_{2}^{*}=-\left[\left(\frac{1}{2}\right) \mathbf{M}_{2}: \mathbf{W}_{2}+\mathcal{W}\right] .
\end{aligned}
$$

Equation (23) states that the rate of work done on constituents by the internal couples contributes to the constituent internal energy as does the contribution arising from the relative motions.

\subsection{Entropy Tendency}

Although the non-negative generation of entropy for mixtures is fundamental to any theory, there is no universal agreement on how it is achieved. Some workers treat this from the standpoint of small deviations from equilibrium as, for example, in [35]. Recently Grmela has proposed a general approach utilizing Hamiltonian mechanics that is less restrictive. See [36,37] and references cited therein.

For present purposes it is sufficient to start with generic partial entropy tendency equations for both constituents based on classical irreversible thermodynamics. In their equation (2.48) Rajagopal and Tao [22] state the sum of these equations, from which it is straightforward to obtain the individual equations in our notation as

$$
\begin{aligned}
& \rho_{1} \frac{d_{1} s_{1}}{d t}+\nabla \cdot \mathbf{J}_{1}-\rho_{1} r_{s 1}=\sigma_{1} \\
& \rho_{2} \frac{d_{2} s_{2}}{d t}+\nabla \cdot \mathbf{J}_{2}-\rho_{2} r_{s 2}=\sigma_{2} .
\end{aligned}
$$

Here the $\mathbf{J}_{\alpha}$ are the transports of $s_{\alpha}$ by non-advective or diffusive processes, the $r_{s \alpha}$ terms account for external entropy supply, and $\sigma_{\alpha}$ are the internal productions of entropy. If thermally interacting materials equations of the form of (4) apply, it is possible that the temperature or velocity gradients of one constituent could be strong enough to produce a negative entropy production for the second constituent. Then $\sigma_{\alpha}<0$ for that constituent. Nevertheless, for the mixture we require

$$
\sigma_{1}+\sigma_{2}=\Gamma \geq 0
$$

The presence of disparate constituent temperatures complicates the analysis of the mixture entropy production terms since there is no consensus definition for the internal energy for a mixture when the components have distinct temperatures. We avoid this issue and follow Eckart [29], de Groot and Mazur [38], Jou et al. [6], and more recently Klika [20] and use the Gibbs relation for the mixture. This presupposes the existence of both entropy and temperature functions. Of course the downside of this approach is that the Gibbs relation applies to equilibrium conditions. This restriction is not critical here as our primary goal is to explore the consequences of the second law on phenomenological parameters. 
Nevertheless the disparate temperatures of the constituents require special consideration. First we apply the Gibbs relation to each constituent of (19) and (24) so as to accommodate the temperatures. Then we sum the resulting equations to give the Gibbs equation for the mixture as

$$
\rho_{1} \frac{d_{1} s_{1}}{d t}+\rho_{2} \frac{d_{2} s_{2}}{d t}=\theta_{1}^{-1}\left(\rho_{1} \frac{d_{1} u_{1}}{d t}-p_{1} \frac{d_{1} v_{1}}{d t}\right)+\theta_{2}^{-1}\left(\rho_{2} \frac{d_{2} u_{2}}{d t}-p_{2} \frac{d_{2} v_{2}}{d t}\right)
$$

In (26) the $p_{\alpha}$ are thermodynamic partial pressures associated with reversible processes, i.e., the pressure each constituent would have if it occupied the mixture space. The sum of the partial pressures would be the mixture partial pressure. Also in (26) the $v_{\alpha}\left(=\rho_{\alpha}^{-1}\right)$ are the partial volumes, and the $\theta_{\alpha}$ are the temperatures of the respective constituents.

Using (23) for the internal energy sources and (13) to express the partial volume changes of the constituents in terms of the respective velocity divergences, (26) is rewritten as

$$
\begin{aligned}
& \rho_{1} \frac{d_{1} s_{1}}{d t}+\rho_{2} \frac{d_{2} s_{2}}{d t}-\theta_{1}^{-1}\left[\rho_{1} \frac{d_{1} u_{1}}{d t}+p_{1} \nabla \cdot \mathbf{v}_{1}\right]-\theta_{2}^{-1}\left[\rho_{2} \frac{d_{2} u_{2}}{d t}+p_{2} \nabla \cdot \mathbf{v}_{2}\right] \\
& =\Gamma-\nabla \cdot\left(\mathbf{J}_{1}+\mathbf{J}_{2}\right)+\nabla \cdot\left(\frac{\mathbf{q}_{1}}{\theta_{1}}+\frac{\mathbf{q}_{2}}{\theta_{2}}\right)+\rho_{1} r_{s 1}+\rho_{2} r_{s 2}+\theta_{1}^{-1} \rho_{1} r_{1}+\theta_{2}^{-1} \rho_{2} r_{2} \\
& -\theta_{1}^{-1}\left[\mathbf{T}_{1}^{T}: \mathbf{D}_{1}+\mathbf{q}_{1} \cdot \nabla \theta_{1}^{-1}-\left(\frac{1}{2}\right) \mathbf{M}_{1}: \mathbf{W}_{1}-\mathcal{W}\right] \\
& -\theta_{2}^{-1}\left[\mathbf{T}_{2}^{T}: \mathbf{D}_{2}+\mathbf{q}_{2} \cdot \nabla \theta_{2}^{-1}-\left(\frac{1}{2}\right) \mathbf{M}_{2}: \mathbf{W}_{2}-\mathcal{W}\right] .
\end{aligned}
$$

The Gibbs formulation given in (27) suggests the mixture entropy flux and mixture external entropy source is given by

$$
\begin{aligned}
& \mathbf{J}_{1}+\mathbf{J}_{2}=\left(\frac{\mathbf{q}_{1}}{\theta_{1}}+\frac{\mathbf{q}_{2}}{\theta_{2}}\right) \\
& \rho_{1} r_{s 1}+\rho_{2} r_{s 2}=\left(\frac{\rho_{1} r_{1}}{\theta_{1}}+\frac{\rho_{2} r_{2}}{\theta_{2}}\right) .
\end{aligned}
$$

It then follows from (27) that

$$
\begin{aligned}
& \Gamma=\left(\theta_{1}^{-1} p_{1} \nabla \cdot \mathbf{v}_{1}+\theta_{2}^{-1} p_{2} \nabla \cdot \mathbf{v}_{2}\right) \\
& +\theta_{1}^{-1}\left[\mathbf{T}_{1}^{T}: \mathbf{D}_{1}+\mathbf{q}_{1} \cdot \nabla \theta_{1}^{-1}-\left(\frac{1}{2}\right) \mathbf{M}_{1}: \mathbf{W}_{1}-\mathcal{W}\right] \\
& +\theta_{2}^{-1}\left[\mathbf{T}_{2}^{T}: \mathbf{D}_{2}+\mathbf{q}_{2} \cdot \nabla \theta_{2}^{-1}-\left(\frac{1}{2}\right) \mathbf{M}_{2}: \mathbf{W}_{2}-\mathcal{W}\right] \geq 0 .
\end{aligned}
$$

Equation (29) is the Clausius-Duhem equation for a two constituent mixture with different temperatures.

\section{Constitutive Equations}

\subsection{Theory}

The main objective of constitutive modeling is to supply connections between kinematical, mechanical, chemical, electro-magnetic, and thermal fields in such a way that when used along with the governing conservation equations the result is a well-posed theory for properly defined problems. Since the fluxes and the dependent variables of the governing equations constitute more unknowns than governing equations, it is necessary to develop constitutive relations between the fluxes and dependent variables so that the number of fundamental equations equals the number of unknowns. Here we employ an additional condition on the constitutive equations: the constitutive 
variables must also be constrained by the second law of thermodynamics. If they do not appear in the second law inequality then they should not be used in the constitutive equations. As noted below this is actually a constraint on the mathematical structure of the appropriate constitutive equations.

We speculate that for a two-constituent mixture of two fluids whose constituents have different temperatures, densities, velocities, etc., the constitutive relations for the stress tensors and the heat flux vectors should depend on the velocity difference, velocity gradient, temperatures, temperature gradient, densities, density gradient, etc. Then application of frame-indifference results in the following objective quantities:

$$
\mathcal{G}^{*}=\left(\rho_{1}, \rho_{2}, \theta_{1}, \theta_{2}, \triangle, \nabla \rho_{1}, \nabla \rho_{2}, \nabla \theta_{1}, \nabla \theta_{2}, \mathbf{a}, \mathbf{D}_{1}, \mathbf{D}_{2}\right) .
$$

Here $\triangle=\theta_{1}-\theta_{2}$ and $\mathbf{a}=\mathbf{v}_{1}-\mathbf{v}_{2}$. There is an obvious redundancy in specifying both $\theta_{1}, \theta_{2}$ as well as their difference. We include both, as some workers use just the latter. Note also that the partial density dependencies can be replaced by the volume fractions $\phi_{\alpha}$.

Equations (13), (14), and (19) are the balance equations that govern the flow of a two-component mixture with different temperatures. To close this system of equations, it is necessary to prescribe constitutive relations for the stress tensors $\mathbf{T}_{\alpha}$, the momentum interaction force $\mathbf{f}_{I}$, and the heat fluxes $\mathbf{q}_{\alpha}$ in terms of objective variables that arise in the balance equations. Constitutive equations characterize the interactions between the constituents, thus different relations are used for various types of flows such as gas-solid, bubbly liquids, solid-liquid, fluid diffusing through an elastic layer, etc. Regardless of their specific forms, the latter equations are subject to the constraint imposed by (29).

\subsection{Linear Constitutive Equations for a Mixture of Two Fluids}

Here we develop constitutive relations to include heat fluxes for each constituent while neglecting density variations, as our analysis is restricted to linear constitutive equations. We take as the objective variable set a restricted version of $\mathcal{G}^{*}$ :

$$
\mathcal{G}=\left(\nabla \theta_{1}, \nabla \theta_{2}, \mathbf{a}, \mathbf{D}_{1}, \mathbf{D}_{2}\right) .
$$

Consider first constitutive equations for the heat fluxes and the momentum interaction force. The general linear representation for the former are assumed to be

$$
\begin{aligned}
& \mathbf{q}_{1}=\alpha_{1} \nabla \theta_{1}+\alpha_{2} \nabla \theta_{2}+\alpha_{3} \mathbf{a} \\
& \mathbf{q}_{2}=\beta_{1} \nabla \theta_{1}+\beta_{2} \nabla \theta_{2}+\beta_{3} \mathbf{a} .
\end{aligned}
$$

Onsager's reciprocal criteria provides some simplification to (32) by requiring $\alpha_{2}=\beta_{1}$; however, it is not necessary to impose this condition here. Note further that $\alpha_{3} \neq-\beta_{3}$ implies a mixture heat flux even in the absence of thermal gradients. The friction associated with the relative motions of the constituents heats both constituents. This process is not accounted for by the Fourier law.

The phenomenological coefficients in (32) are functions of the invariants $\mathcal{H}$ of $\mathcal{G}$. See [39] for details on invariants of vectors and tensors. Specifically

$$
\begin{aligned}
\alpha_{i} & =\alpha_{i}(\mathcal{H}) \\
\beta_{i} & =\beta_{i}(\mathcal{H}) .
\end{aligned}
$$

Similar to (32) $\mathbf{f}_{I}$ is assumed to be given as

$$
\mathbf{f}_{I}=\gamma_{1} \mathbf{a}+\gamma_{2} \nabla \theta_{1}+\gamma_{3} \nabla \theta_{2}
$$

with $\gamma_{1}, \gamma_{2}$, and $\gamma_{3}$ also functions of $\mathcal{H}$. It is noteworthy that (34) allows for a transfer of momentum between the constituents due to the constituent temperature gradients even in the absence of relative motion of the constituents. 
Müller [34] indicated that in order to get well-posed problems, the interaction forces due to density gradients, i.e., $\nabla \rho_{1}$ and $\nabla \rho_{2}$, should also be included among the interaction forces in (34). For a detailed review and discussion of various interaction forces such as lift forces, forces due to mixing, virtual mass, etc., see [40]. However, in the linear theory considered here there are no second laws constraint on these processes; consequently they are excluded in our analysis.

Now consider constitutive equations for the partial stresses. These are specified as

$$
\begin{aligned}
& \mathbf{T}_{1}=\left(-p_{1}+\lambda_{1} t r \mathbf{D}_{1}+\lambda_{3} t r \mathbf{D}_{2}\right) \mathbf{I}+2 \mu_{1} \mathbf{D}_{1}+2 \mu_{3} \mathbf{D}_{2}+\lambda_{5}\left(\mathbf{W}_{1}-\mathbf{W}_{2}\right) \\
& \mathbf{T}_{2}=\left(-p_{2}+\lambda_{4} t r \mathbf{D}_{1}+\lambda_{2} \operatorname{tr} \mathbf{D}_{2}\right) \mathbf{I}+2 \mu_{4} \mathbf{D}_{1}+2 \mu_{2} \mathbf{D}_{2}-\lambda_{5}\left(\mathbf{W}_{1}-\mathbf{W}_{2}\right) .
\end{aligned}
$$

Here $\mathbf{I}$ is the identity operator.Note also that these equations specify $\mathbf{M}_{1}=-\mathbf{M}_{2}=\lambda_{5}\left(\mathbf{W}_{1}-\mathbf{W}_{2}\right)$. There are a total of 9 phenomenological coefficients in (35), which are functions of $\mathcal{H}$. Application of Onsager's reciprocal relation principle would reduce the number to 7 by setting $\lambda_{3}=\lambda_{4}$ and $\mu_{3}=\mu_{4}$.

\section{Entropy Production Constraints on the Constitutive Equations}

In this section we use the Clausius-Duhem inequality (29) to determine constraints on the phenomenological coefficients in the constitutive equations for a two-fluid mixture given by (32), (34), and (35). They are determined by inserting (32), (34), and (35) into (29). Our approach relies on establishing conditions on the phenomenological coefficients that insure non-negative quadratic forms for the Clausius-Duhem inequality. Since we regard this condition to be a property of the quadratic form, we employ symmetrized forms of the phenonemological matrices. This avoids evoking Onsager conditions on the cross gradient coefficients a-priori, but reduces to that case when these conditions are prescribed aposteriori.

The result can be broken down into entropy production by processes due to vectorial terms $\mathcal{V}$ and tensorial terms $\mathcal{T}$ as

$$
\Gamma=\mathcal{V}+\mathcal{T} \geq 0
$$

Following [41], we expect $\mathcal{V}$ and $\mathcal{T}$ to be $\geq 0$ independently.

Consider first the contribution to entropy production from the vector processes. After some calculation, using (32) and (34) one obtains $\mathcal{V}$ as

$$
\mathcal{V}=\left[\nabla \theta_{1}, \nabla \theta_{2}, \mathbf{a}\right]^{T} \cdot \mathbf{\Lambda} \cdot\left[\nabla \theta_{1}, \nabla \theta_{2}, \mathbf{a}\right] \geq 0
$$

with

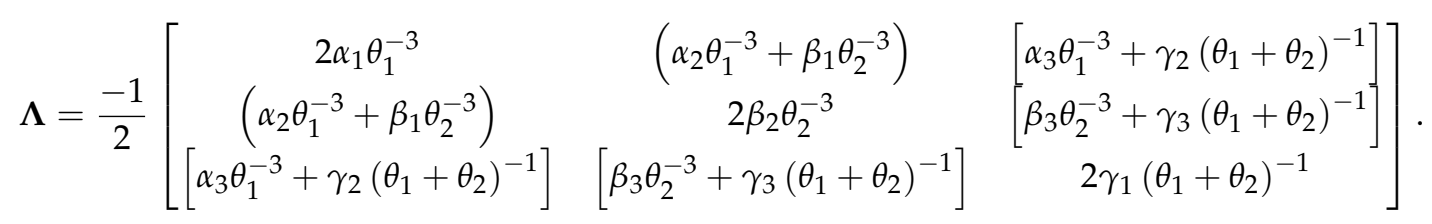

Equation (38) differs from an earlier result given in [26] where the energy source terms were not frame invariant.

The requirement for non-negative entropy production is that $\Lambda$ be non-negative definite. This requires the 6 determinants of the principal submatrices and the determinate of $\Lambda$ to be non-negative definate. Thus

$$
\alpha_{1}, \beta_{2}, \gamma_{1} \leq 0
$$


since $\theta_{1}, \theta_{2}>0$. The inequality constraints arising from the second order principal minors are

$$
\begin{aligned}
& \frac{\alpha_{1} \beta_{2}}{\theta_{1}^{3} \theta_{2}^{3}}-\left(\frac{1}{4}\right)\left(\frac{\alpha_{2}}{\theta_{1}^{3}}+\frac{\beta_{1}}{\theta_{2}^{3}}\right)^{2} \leq 0 \\
& \left(\frac{\alpha_{1}}{\theta_{1}^{2}}\right)\left(\frac{\gamma_{1}}{\theta_{1}+\theta_{2}}\right)-\left(\frac{1}{4}\right)\left(-\frac{\beta_{3}}{\theta_{2}^{3}}+\frac{\gamma_{3}}{\theta_{1}+\theta_{2}}\right)^{2} \leq 0 \\
& \left(\frac{\beta_{2}}{\theta_{2}^{3}}\right)\left(\frac{\gamma_{1}}{\theta_{1}+\theta_{2}}\right)-\left(\frac{1}{4}\right)\left(-\frac{\beta_{3}}{\theta_{2}^{3}}+\frac{\gamma_{3}}{\theta_{1}+\theta_{2}}\right)^{2} \leq 0 .
\end{aligned}
$$

The determinate of $\Lambda$ provides a cubic constraint, but it is not recorded here.

Now consider the second law constraints on the tensor processes. Here

$$
\begin{aligned}
& \mathcal{T}=\left[\lambda_{1}\left(\operatorname{tr} \mathbf{D}_{1}\right)^{2}+\lambda_{3}\left(t r \mathbf{D}_{2}\right)\left(\operatorname{tr} \mathbf{D}_{1}\right)+2 \mu_{1} \operatorname{tr} \mathbf{D}_{1}^{2}+2 \mu_{3} \operatorname{tr}\left(\mathbf{D}_{2} \mathbf{D}_{1}\right)+\lambda_{5} \operatorname{tr}\left[\left(\mathbf{W}_{1}-\mathbf{W}_{2}\right) \mathbf{W}_{1}\right]\right] \theta_{1}^{-1} \\
& +\left[\lambda_{4}\left(\operatorname{tr} \mathbf{D}_{1}\right)\left(\operatorname{tr} \mathbf{D}_{2}\right)+\lambda_{2}\left(\operatorname{tr} \mathbf{D}_{2}\right)^{2}+2 \mu_{4} \operatorname{tr}\left(\mathbf{D}_{1} \mathbf{D}_{2}\right)+2 \mu_{2} \operatorname{tr} \mathbf{D}_{2}^{2}-\lambda_{5} \operatorname{tr}\left[\left(\mathbf{W}_{1}-\mathbf{W}_{2}\right) \mathbf{W}_{2}\right]\right] \theta_{2}^{-1} .
\end{aligned}
$$

Using (29) in (35) it is found the partial pressures $p_{\alpha}$ cancel in obtaining (41). Thus they do not play any role in the generation of entropy for the mixture, [6,29], consequently they are not considered further. Such processes are elegantly treated by Hamiltonian mechanics methods as described in [36,37].

Rendering (41) in matrix form gives

$$
\begin{aligned}
& \mathcal{T}=\left[\operatorname{tr}\left(\mathbf{D}_{1}\right), \operatorname{tr}\left(\mathbf{D}_{2}\right)\right]\left[\begin{array}{cc}
\frac{\lambda_{1}}{\theta_{1}} & \left(\frac{1}{2}\right)\left(\frac{\lambda_{3}}{\theta_{1}}+\frac{\lambda_{4}}{\theta_{2}}\right) \\
\left(\frac{1}{2}\right)\left(\frac{\lambda_{3}}{\theta_{1}}+\frac{\lambda_{4}}{\theta_{2}}\right) & \frac{\lambda_{2}}{\theta_{2}}
\end{array}\right]\left[\begin{array}{l}
\operatorname{tr}\left(\mathbf{D}_{1}\right) \\
\operatorname{tr}\left(\mathbf{D}_{2}\right)
\end{array}\right]
\end{aligned}
$$

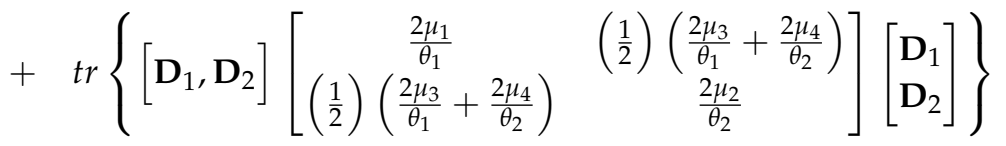

$$
\begin{aligned}
& +\lambda_{5} \operatorname{tr}\left\{\left[\mathbf{W}_{1}, \mathbf{W}_{2}\right]\left[\begin{array}{cc}
\frac{1}{\theta_{1}} & -\left(\frac{1}{2}\right)\left(\frac{1}{\theta_{1}}+\frac{1}{\theta_{2}}\right) \\
-\left(\frac{1}{2}\right)\left(\frac{1}{\theta_{1}}+\frac{1}{\theta_{2}}\right) & \frac{1}{\theta_{2}}
\end{array}\right]\left[\begin{array}{l}
\mathbf{W}_{1} \\
\mathbf{W}_{2}
\end{array}\right]\right\} \text {. }
\end{aligned}
$$

Consider the first term on the right-hand side of (42). Note that this is a quadratic form of $\operatorname{tr} \mathbf{D}_{1}, \operatorname{tr} \mathbf{D}_{2}$. The requirement that this form be non-negative is

$$
\begin{aligned}
& \frac{\lambda_{1}}{\theta_{1}} \geq 0 \\
& \frac{\lambda_{2}}{\theta_{2}} \geq 0 \\
& \frac{\lambda_{1} \lambda_{2}}{\theta_{1} \theta_{2}}-\left(\frac{\lambda_{3}}{\theta_{1}}+\frac{\lambda_{4}}{\theta_{2}}\right)^{2} \geq 0 .
\end{aligned}
$$

Similarly, the second term in (42) is a quadratic form of $\operatorname{tr}\left(\mathbf{D}_{1}^{2}, \mathbf{D}_{2}^{2}\right) \geq 0$. From this it is concluded that

$$
\begin{aligned}
& \frac{\mu_{1}}{\theta_{1}} \geq 0 \\
& \frac{\mu_{2}}{\theta_{2}} \geq 0 \\
& \frac{\mu_{1} \mu_{2}}{\theta_{1} \theta_{2}}-\left(\frac{1}{4}\right)\left(\frac{2 \mu_{3}}{\theta_{1}}+\frac{2 \mu_{4}}{\theta_{2}}\right)^{2} \geq 0 .
\end{aligned}
$$


The third line of (42) is a quadratic form in $\mathbf{W}_{1}$ and $\mathbf{W}_{2}$. Since the matrix determinate is non-positive the relevant constraint requiring non-negative entropy production is

$$
\lambda_{5} \leq 0 .
$$

These inequalities reduce to the standard condition for a linear Navier-Stokes fluid when the temperatures of the two constituents are the same.

The main results of this section are the constraints imposed by the second law on the coefficients in the constitutive equations. These are equations (39), (40), (43), (44) and (45). After adjusting for notation, these results are the same as reported by those earlier in [26]. Note that if $\theta_{1}=\theta_{2}$ these constraints reduce to well-established conditions. The interesting aspect is the role the constituent temperatures play in the second order principal minor constraints. These could be relevant to experimentalists and to the stability of solutions of specific problems.

\section{Special Cases}

\subsection{Heat Flux}

We are now in a position to study several special cases of the constitutive equations for heat flux vectors and the interaction force $\mathbf{f}_{I}$ that are relevant to the issues raised in Section 1. First, recall (32)

$$
\begin{aligned}
& \mathbf{q}_{1}=\alpha_{1} \nabla \theta_{1}+\alpha_{2} \nabla \theta_{2}+\alpha_{3} \mathbf{a} \\
& \mathbf{q}_{2}=\beta_{1} \nabla \theta_{1}+\beta_{2} \nabla \theta_{2}+\beta_{3} \mathbf{a} .
\end{aligned}
$$

The first two terms are a generalization of the Fourier law of heat conduction that accounts for constituent heat fluxes that depend on the temperature gradients in both constituents. The third term predicts a contribution to the constituent heat fluxes from friction arising from the different motions of the constituents. However, if $\alpha_{3}=-\beta_{3}$ this term cancels in $\mathbf{q}_{\text {mix }}$ and so the result is (5). If further $\theta_{1}=\theta_{2}$, then

$$
\mathbf{q}_{\text {mix }}=\left(\alpha_{1}+\alpha_{2}+\beta_{1}+\beta_{2}\right) \nabla \theta .
$$

Hence $\alpha_{1}+\alpha_{2}+\beta_{1}+\beta_{2}=k$ is the effective thermal conductivity of a mixture as noted in (3). Moreover, q obtained from (46) is the same form as equation (6.39) in [31], who wrote this as

$$
\mathbf{q}=\alpha \nabla \theta+\alpha_{i k}\left(\mathbf{v}_{i}-\mathbf{v}_{k}\right) .
$$

Note also that the momentum transfer between constituents simplifies to

$$
\mathbf{f}_{I}=\gamma_{1} \mathbf{a}+\left(\gamma_{2}+\gamma_{3}\right) \nabla \theta .
$$

\subsection{Green Adkins Massoudi Theory}

In response to the ontological question "How does mixture know it is a mixture?" Truesdell [21] proposed three metaphysical principles to govern mixture dynamics. Although widely accepted, they apparently do not account for all mixture behavior. Consequently several additional metaphysical principles have been proposed. A recent review is given in [26]. Here we investigate the Green Adkins Massoudi principle. Simply stated, this principle requires the governing equations and the constitutive relations for mixtures to reduce to the appropriate form for a single constituent if the concentrations of the other constituents vanish. This can be achieved by a number of weighting functions. Here we elect to follow Massoudi, [33], by requiring the phenomenological coefficients to be functions of the volume fractions of the constituents. That choice is also consistent with the analysis given in $[27,42]$. See also a recent discussion of volume fractions in [20]. 
Application to a fluid-fluid mixture with the phenomenological coefficients in (32), (34), and (35) taken as linear functions of the volume fraction $\phi$ of constituent 2 gives

$$
\begin{aligned}
\mathbf{q}_{1} & =(1-\phi)\left[\alpha_{30} \nabla \theta_{1}+\phi \alpha_{40} \nabla \theta_{2}+\phi \alpha_{50} \mathbf{a}\right] \\
\mathbf{q}_{2} & =\phi\left[(1-\phi) \beta_{30} \nabla \theta_{1}+\beta_{40} \nabla \theta_{2}-(1-\phi) \alpha_{50} \mathbf{a}\right] \\
\mathbf{f}_{I} & =\phi(1-\phi)\left(\gamma_{10} \mathbf{a}+\gamma_{20} \nabla \theta_{1}+\gamma_{30} \nabla \theta_{2}\right) \\
\mathbf{T}_{1} & =(1-\phi)\left[\left(\lambda_{10} \operatorname{tr} \mathbf{D}_{1}+\phi \lambda_{30} \operatorname{tr} \mathbf{D}_{2}\right) \mathbf{I}+2\left(\mu_{10} \mathbf{D}_{1}+\phi \mu_{30} \mathbf{D}_{2}\right)+\phi \lambda_{50}\left(\mathbf{W}_{1}-\mathbf{W}_{2}\right)\right] \\
\mathbf{T}_{2} & \left.=\phi\left\{\left[(1-\phi) \lambda_{4} \operatorname{tr} \mathbf{D}_{1}+\lambda_{2} \operatorname{tr} \mathbf{D}_{2}\right] \mathbf{I}+2\left[(1-\phi) \mu_{40}\right) \mathbf{D}_{1}+\mu_{2} \mathbf{D}_{2}\right]\right\} \\
& -\phi(1-\phi) \lambda_{50}\left(\mathbf{W}_{1}-\mathbf{W}_{2}\right) .
\end{aligned}
$$

It is stressed that (49) reduces to the standard constitutive equations for a Navier-Stokes fluid in the extreme cases of $\phi=1,0$. Moreover, the inclusion of the volume fraction in the phenomenological coefficients in (49) does not impact the thermodynamic constraints given by equations (39), (40), (43), (44) and (45); see [26].

The mixture heat flux implied by (49) is

$$
\mathbf{q}_{\text {mix }}=(1-\phi)\left(\alpha_{30}+\phi \beta_{30}\right) \nabla \theta_{1}+\phi\left[(1-\phi) \alpha_{40}+\beta_{40}\right] \nabla \theta_{2} .
$$

The heat flux generated by the differential movement of the two constituents does not contribute to $\mathbf{q}_{\text {mix }}$, and the conductivities are weighted by the respective volume fractions. Moreover, when $\nabla \theta_{1}=\nabla \theta_{2}$ the effective conductivity includes contributions from the cross flux terms $\alpha_{40}$ and $\beta_{30}$. These, however, are weighted by $\phi(1-\phi)$ and further constrained by (40).

From (49) a mixture stress can be defined as

$$
\mathbf{T}_{m i x}=\mathbf{T}_{1}+\mathbf{T}_{2}
$$

The weighting system in (51) is similar to that proposed in [31,32] for the mixture stress, except that it includes the cross viscous terms $\mu_{30}$ and $\mu_{40}$ weighted by the respective volume fractions.

\section{Conclusions}

Several new results have emerged from this effort. First, we extended mixture theory for two Navier-Stokes fluids to include temperature differences between the constituents. The general theory has 18 phenomenological coefficients. This number can be reduced to 15 if Onsager's reciprocal principle is evoked. Despite this rather large number, constraints on the constitutive equations were established by the requirement of non-negative entropy production and shown to be consistent with previous results.

Secondly, consider the constitutive model given by (49). As suggested by Hansen et al., [27] volume fraction serves as a weighting function for the constituent stresses and heat fluxes in the same fashion Truesdell [21] proposed using constituent densities to define the mixture velocity. That paper also provides a succinct argument for using volume fraction for these fluxes.

Thirdly, the heat flux laws proposed here will require new phenomenological parameters and thus pose significant challenges to experimentalists. Weighting the constitutive equations by the constituent volume fractions or other suitable weighting function could be an important attribute in this regard since some of the coefficients can be determined from experiments with pristine substances. The cross gradient fluxes, of course, will remain a challenge for experimentalists. However, the second law inequalities established here put useful constraints on their values.

Although the constitutive models considered here are for two fluids, it has some applicability to the mixture of a fluid and a solid. As an example, consider the two limiting cases of (49). In the first case, suppose $\phi \rightarrow 0$, i.e., the particle component vanishes. Then $\alpha_{1}=k_{1}$, indicating that we have a case of pure fluid. In the second case, $\phi \rightarrow 1$, i.e., the fluid component vanishes and we have a solid matrix, $\beta_{1}=k_{2}$, where $k_{2}$ is the pure thermal conductivity of the solid material. 
This study calls attention to other areas for further investigation. First is the energy equation for mixtures with different constituent temperatures. The case of equal constituent temperatures is straightforward and well established in the literature, whereas the two-temperature scenario considered here is not. A second area involves extending the theory to include density effects such as in suspensions of granular materials in fluids as studied by [43-45]. Here the constitutive equations will include second order effects such as density gradients in the stress constitutive equations. This means the entropy inequality will no longer be of quadratic form. Nevertheless, as shown in $[44,46]$, second law constraints on the phenomenological coefficients can still be obtained.

Author Contributions: Conceptualization, A.D.K.J. and M.M.; Formal analysis, A.D.K.J. and M.M.; Investigation, A.D.K.J. and M.M.; Methodology, A.D.K.J. and M.M.; Validation, A.D.K. and M.M.; Writing: original draft, A.D.K.J. and M.M.; Writing: review \& editing, A.D.K.J. and M.M. Both authors contributed equally to the analysis and writing of the manuscript. All authors have read and agreed to the published version of the manuscript.

Funding: This research received no external funding.

Acknowledgments: The authors gratefully acknowledge a reviewer's suggestions for making the constituent energy and entropy equations frame invariant. Karal Gregory supplied outstanding technical support in preparation of the manuscript.

Conflicts of Interest: The authors declare no conflict of interest.

\section{References}

1. Dunwoody, N.T.; Müller, I.A. A thermodynamic theory of two chemically reacting ideal gases with different temperatures. Arch. Ration. Mech. Anal. 1968, 29, 344-369. [CrossRef]

2. Bowen, R.M.; Garcia, D.J. On the thermodynamics of mixtures with several temperatures. Int. J. Eng. Sci. 1970, 8, 63-83. [CrossRef]

3. Ahmadi, G. Thermodynamics of multi-temperature fluids with applications to turbulence modelling. Appl. Math. Model. 1985, 9, 271-274. [CrossRef]

4. Maxwell, J.C. On the dynamic theory of gases. Philos. Trans. R. Soc. Lond. 1867, 147, 49-88.

5. Cattaneo, C. Sulla Conduzione Del Calore. Atti Semin. Mat. Fis. Univ. Modena 1948, 3, 83-101.

6. Jou, D.; Casas-Vazquez, J.; Lebon, G. Extended Irreversible Thermodynamics. In Extended Irreversible Thermodynamics; Springer: Berlin, Germany, 1996; pp. 41-74.

7. Petroski, H.J. Departures from Fourier's law. ZAMP 1975, 26, 119-124. [CrossRef]

8. Mitchell, J.K. Conduction phenomena: From theory to geotechnical practice. Geotechnique 1991, 41, $299-340$. [CrossRef]

9. Bashir, Y.M.; Goddard, J.D. Experiments on the conductivity of suspensions of ionically conductive spheres. AlChE J. 1990, 36, 387-396. [CrossRef]

10. Prasher, R.S.; Koning, P.; Shipley, J.; Devpura, A. Dependence of thermal conductivity and mechanical rigidity of particle-laden polymeric thermal interface material on particle volume fraction. ASME J. Electron. Packag. 2003, 125, 386-391. [CrossRef]

11. Lee, D.L.; Irvine, T.F., Jr. Shear rate dependent thermal conductivity measurements of non-Newtonian fluids. Exp. Therm. Fluid Sci. 1997, 15, 16-24. [CrossRef]

12. Massoudi, M. On the heat flux vector for flowing granular materials, Part 1: Effective thermal conductivity and background. Math. Methods Appl. Sci. 2006, 29, 1585-1598. [CrossRef]

13. Massoudi, M. On the heat flux vector for flowing granular materials, Part 2: Derivation and special cases. Math. Methods Appl. Sci. 2006, 29, 1599-1613. [CrossRef]

14. Massoudi, M. On the heat flux vector in mixtures. Int. Commun. Heat Mass Transf. 2005, 32, 1111-1266. [CrossRef]

15. Klika, V.; Pavelka, M.; Benziger, J.B. Functional constraints on phenomenological coefficients. Phys. Rev. E 2017, 95, 022125. [CrossRef]

16. Klika, V.; Krause, A.L. Beyond Onsager-Casimir relations: Shared dependence of phenomenological coefficients on state variables. J. Phys. Chem. Lett. 2018, 9. [CrossRef] 
17. Rajagopal, K.R.; Srinivasa, A.R. A thermodynamic frame work for rate type fluid models. J. Non-Newtonian Fluid Mech. 2000, 88, 207-227. [CrossRef]

18. Rajagopal, K.R.; Srinivasa, A.R. Modeling anisotropic fluids within the framework of bodies with multiple natural configurations. J. Non-Newtonian Fluid Mech. 2001, 99, 109-124. [CrossRef]

19. Rajagopal, K.; Srinivasa, A. On the development of fluid models of the differential type within a new thermodynamical framework. Mech. Res. Commun. 2008. doi:10.1016/j.mechrescom.2008.02.004. [CrossRef]

20. Klika, V. A guide through available mixture theories for applications. Crit. Rev. Solid State Mater. Sci. 2014, 39, 154-174. [CrossRef]

21. Truesdell, C. Rational Thermodynamics, 2nd ed.; Springer: New York, NY, USA, 1984.

22. Rajagopal, K.R.; Tao, L. Mechanics of Mixtures; World Scientific: Singapore, 1995.

23. Atkin, R.J.; Craine, R.E. Continuum theories of mixtures: Applications. IMA J. Appl. Math 1976, 17, $153-207$. [CrossRef]

24. Atkin, R.J.; Craine, R.E. Continuum theories of mixtures: Basic theory and historical development. Q. J. Mech. Appl. Math. 1976, 29, 209-244. [CrossRef]

25. Bowen, R.M. Theory of Mixtures. In Continuum Physics; Eringen, A.C., Ed.; Academic Press: New York, NY, USA, 1976; Volume 3, pp. 1-127.

26. Kirwan, A.D., Jr. Second Law constraints on the dynamics of a mixture of two fluids at different temperatures. Entropy 2012, 14, 880-891. [CrossRef]

27. Hansen, A.C.; Crane, R.L.; Damson, M.H.; Donovan, M.H.; Horning, R.P.; Walker, J.L. Some notes on a volume fraction mixture theory and a comparison with the kinetic theory of gases. Int. J. Eng. Sci. 1991, 29, 561-573. [CrossRef]

28. Beevers, C.E.; Craine, R.E. On the determination of response functions for a binary mixture of incompressible Newtonian fluids. Int. J. Eng. Sci. 1982, 20, 737-745. [CrossRef]

29. Eckart, C. The thermodynamics of irreversible processes, II Fluid mixtures. Phys. Rev. E 1940, 58, $269-275$. [CrossRef]

30. Truesdell, C.; Noll, W. The Classical Field Theories; Springer: Berlin, Germany, 1965; Volume 3.

31. Green, A.E.; Naghdi, P.M. A theory of mixtures. Arch. Ration. Mech. Anal. 1967, 24, 243-263. [CrossRef]

32. Green, A.E.; Naghdi, P.M. A note on mixtures. Int. J. Eng. Sci. 1968, 6, 631-635. [CrossRef]

33. Massoudi, M. A note on the meaning of mixture viscosity using the classical continuum theories of mixtures. Int. J. Eng. Sci. 2008, 46, 677-689. [CrossRef]

34. Müller, I.A. A thermodynamic theory of mixtures of fluids. Arch. Ration. Mech. Anal. 1968, 28, 1-39. [CrossRef]

35. Dunn, J.E.; Serrin, J. On thermodynamics of interstitial working. Arch. Ration. Mech. Anal. 1985, 88, 95-133. [CrossRef]

36. Grmela, M. Externally driven macroscopic systems: Dynamics versus thermodynamics. J. Stat. Phys. 2017, 166, 282-316. [CrossRef]

37. Grmela, M. Generic guide to the multiscale dynamics and thermodynamics. J. Phys. Commun. 2018, 2, 032001. [CrossRef]

38. de Groot, S.; Mazur, P. Non-Equilibrium Thermodynamics; Dover Publications, Inc.: New York, NY, USA, 1984.

39. Zheng, Q.S. Theory of representations for tensor functions-A unified invariant approach to constitutive equations. Appl. Mech. Rev. 1994, 47, 545-587. [CrossRef]

40. Massoudi, M. Constitutive relations for the interaction force in multicomponent particulate flows. Int. J. Non-Linear Mech. 2003, 38, 313-336. [CrossRef]

41. Kuiken, G.D.C. Thermodynamics of Irreversible Processes; Wiley: New York, NY, USA, 1994.

42. Klika, V.; Whiteley, J.P.; Brown, C.P.; Gaffney, E.A. The combined impact of tissue heterogeneity and fixed charge for models of cartilage: The one-dimensional biphasic swelling model revisited. Biomech. Model. Mechanobiol. 2019, 18, 953-968. [CrossRef]

43. Yang, H.; Massoudi, M. Conduction and convection heat transfer in a dense granular suspension. Appl. Math. Comput. 2018, 332, 351-362. [CrossRef]

44. Yang, H.; Massoudi, M.; Kirwan, A. D., Jr. Entropy analysis for a nonlinear fluid with a nonlinear heat flux vector. Entropy 2017, 19, 689. [CrossRef] 
45. Massoudi, M. On the flow of granular materials with variable material properties. Int. J. Non-Linear Mech. 2001, 36, 25-37. [CrossRef]

46. Massoudi, M.; Kirwan, A. D., Jr. On the thermodynamics of a nonlinear heat conducting suspension. Fluids 2016, 1, 19. [CrossRef] 